\title{
Broadening Relevance: The Polemic Property of Concepts in Metaphorical Language
}

\section{ESJ Humanities}

\section{Salim Bouherar}

University of Mohamed El Bachir El Ibrahimi, Bordj Bou Arreridj, Algeria

\section{Ramona Pistol}

University of Northampton, UK

Submitted: 15 November 2020

Accepted: 04 December 2020

Published: 31 December 2020

Corresponding author:

Salim Bouherar

DOI: 10.19044/esj.2020.v16n35p19

(c) Copyright 2020 Bouherar S. \& Pistol R. Distributed under Creative Commons BY-NC-ND 4.0 OPEN ACCESS

\begin{abstract}
The lexical pragmatic processes that relevance theory heavily relies on to account for communication are problematic. Broadening and narrowing no longer seem suitable to explain how "conceptual" meanings are modified in the comprehension processes if considering recent developments around the concept definitions and how the mind works. Using theoretical suggestions in psycholinguistics to shed light on the issue raised, the paper makes two main suggestions. Firstly, the account of concepts in relevance theory should be reconsidered to allow for more flexibility. An account of metaphors based on pragmatic adjustment of one of its constituents under the influence of another, as well as contextual assumption, does not seem a viable mental process involved in metaphor comprehension since it should not only allow flexibility of semantic associations but also a reconsideration of concepts as entities and encyclopaedical information as mere conceptual characteristics. Secondly, more cultural awareness and influence should be integrated into the relevance theoretical account of meaning since cultural traits are often considered by speakers in reaching relevance. Moreover, pre-existing conceptual knowledge varies greatly between individuals and thus the comprehension of metaphor should no longer be treated as a static process but rather a highly dynamic one.
\end{abstract}

Keywords: Relevance theory, metaphors, idioms, concepts, cultural assumptions, representations 


\section{Introduction}

The growing body of research in cognition and metaphor processing offers different approaches and views. Within a relevance-theoretic framework (Sperber and Wilson, 1995), a modular approach was set to replace Grice's thoughts about understanding figures of speech. For instance, the metaphor My lawyer is a shark is considered a clear violation of the maxim of quality (example discussed at length in Vega-Moreno, 2005; a similar example also discussed by Clark, 2013), and therefore the hearer is believed to take different routes of comprehension after realising that the attribute of shark to a lawyer need to be understood figuratively and not literally since such a model of comprehension would lead the hearer to nowhere. However, according to relevance theory, the processing is inferential and dependent on narrowing and broadening of concepts (Carston, 2015; Wilson and Carston, 2007; Wilson and Sperber, 2004).

Notwithstanding the rational of relevance theory to interpret figures of speech seems logical, some weaknesses need to be discussed with regards to metaphorical utterances comprehension. There seem to be key issues with formulated ad hoc concepts through lexical adjustments of constituents and the encyclopaedic assumptions that lead to their formulation. Discussing concepts and addressing them from different angles implies that lexical adjustments are flawed.

The paper aims to examine why and how concepts are used within the relevance-theoretic framework to account for metaphors and idioms and then to look at cultural elements which should be considered in determining meaning. Firstly, the paper provides a short overview of the "deflationary" view of metaphors in relevance theory (Sperber and Wilson, 2008), heavily based on a propositional view of communication. Secondly, the paper discusses such a rigidity looking at the issues caused by concept modulation process involved in metaphors and idioms. Thirdly, it considers cross-cultural comprehension, an area which should be addressed in a comprehensive theoretical model of communication. Fourthly, it addresses the rigidness of the metaphorical processing claims when trying to accommodate cultural variations of meaning.

The present study is a secondary research using existing theoretical frameworks of metaphors which provide a wealth of data to help with a broader understanding of the nature of metaphors. The paper looks at theories of figurative language from mainly psycholinguistics accounts which focus on mental processes in the production and comprehension of language. Additionally, the paper looks at mainly two theories (conceptual metaphor theory and relevance theory), which despite several opposing arguments, they also share similarities and both have greatly contributed to the ongoing debate on metaphors and idioms. This paper is a theory-based discussion challenging 
relevance theory accounts on the comprehension of figurative aspects of speech. The psycholinguistic theoretical accounts provide a solid ground for the discussion that appear in this paper with specific interest in the comprehension and the processing of idioms and metaphors within a relevance-theoretic framework of Carston, Wilson, Sperber, and Fodor writings mainly.

The merely cognitive aspects of the theory do not unveil how processing takes place under culturally biased contexts and the novelty of metaphoric utterances within culturally dependent contextual effects. This paper suggests that the principle of relevance should, arguably, be extended to include more than just the modulated aspects of concepts, if there are any as presented by relevance theorists. It also explores more routes of comprehension related to idioms and metaphors, in particular, which regard cross-cultural communication that uses different cultural assumptions with requisite lexical adjustments. This research does not suggest applicable alternatives but rather points to the fields that need to be carefully looked at and reconsidered when discussing figures of speech comprehension. More research is needed to further suggest an adjusted version of the relevancetheoretic account that can explain cognitive metaphorical aspects to be more rhetoric.

\section{The relevance theoretical account of metaphors and idioms}

Building on the work of Grice (1975), relevance theory (Sperber and Wilson, 1995; Wilson and Sperber, 2004; Carston, 2002) can be commended for introducing the concept of "relevance" in communication, a property of inputs to cognitive process which allows us to represent information mentally and make inferences. In the relevance-theoretical framework, all communication results from people's search for and maximisation of relevance (Sperber and Wilson, 2002) to have enough related conclusions with the minimum mental effort to arrive at an accepted comprehension level. Thus, metaphor comprehension is based on the standard relevance-based inferential process of following the path of least effort in testing interpretation until expectation of relevance is satisfied.

While many other theoretical frameworks consider metaphors to be a special and distinct phenomenon (see Lakoff and Johnson, 1980; Bowdle and Gentner, 2005; Glucksberg and Keysar, 1990; Steen 2011), relevance theory stands out as a theoretical framework which offers an account where metaphor is considered similar to any other language type interpretations. The creators of the theory, Dan Sperber and Deirdre Wilson strongly argue that "there is no mechanism specific to metaphors, and no interesting generalization that applies only to them" (Sperber and Wilson, 2008, p.84). Just like many other instances of loose uses of language such as hyperbole, metaphor is placed on 
a continuum with mundane non-figurative loose uses such as approximations and category extensions. Metaphor results from the processes that hearers use linguistic and contextual clues to change a concept from having obstinate characteristics to more compliant with the characteristics of other related/unrelated concepts to the ones verbally expressed. That process is known as pragmatical modulation and it results in ad-hoc (occasion-specific) concepts which contribute to the proposition explicitly communicated (the explicature in relevance theoretical terminology), as well as communicating a range of implicatures (assumptions communicated implicitly) (Carston, 2002; Vega-Moreno, 2007; Wilson and Carston, 2007; Wilson and Sperber, 2004). For example, people understand the metaphor (1) My job is a jail by automatically adjusting the lexical concept JAIL and creating an ad-hoc concept JAIL* through a relevance driven process that uncovers the speaker's informative intention. The assumptions in metaphorical understanding vary, making possible for different interpretations. Therefore, in relevance theory metaphor is simply a case of using the inferential mechanisms of concept adjustment, just like any other interpretations (Sperber and Wilson, 2008).

Also, relevance theory is grounded in a propositional and conceptual view of cognition which leads to the assumption that an utterance communicates a proposition, which is a mental representation in a conceptual format, and this expresses a thought. A direct implication is that relevance theory does not distinguish between the information the brain receives from perception, imagery and other cognitive processes. Compared to embodied approaches such as Conceptual Metaphor Theory (Lakoff and Johnson, 1980) where imagery is a mental representation, the relevance-theoretic fully propositional account does not allow for mental images to have special properties of their own. Moreover, the account distinguishes between two levels of propositional content: the explicature and implicature (Carston and Hall, 2012) and only recently proposed ways to integrate non-propositional (feelings, impressions, emotions) aspects, which can no longer be ignored when talking about figurative language. For example, even when using a conventional metaphor such as

(2) Life is a roller-coaster

or an idiomatic expression such as

(3) He is as good as a one-legged man in a butt-kicking contest, it is almost impossible not to address the impressions that these expressions bring into the context and which might greatly contribute to comprehension. Golding (2015) points out that the propositional view of cognition presented by relevance theorists would benefit from incorporating an embodied view of the mind to include perceptual information such as mental imagery in the determination of speaker meaning. This research agrees and suggests an analogical reasoning process related to perception. Because a propositional 
account such as relevance theory works with representations of logical relationships and propositions, the mechanisms involved in imagery and perception are great challenges in explaining metaphors.

Despite a very high number of studies which have addressed metaphor from several angles, the phenomenon is still perceived as a unique in communication studies (see the second-generation approach to cognitive studies). Relevance theory remains a significant theoretical perspective which has drawn attention to the importance of context in metaphorical studies. However, a thoughtfully detailed explanation of the contribution of contextual features on how metaphors are comprehended is still needed because of the close relationship of meaning to the context. Even a conventional metaphor such as example (1) also mentioned above

(1) My job is a jail

is highly context-sensitive, resulting in two different metaphorical meanings; it can either refer to being watched and lacking autonomy in a context when the previous discussion centres around the speaker's feeling of being continually monitored or it can refer to a prisoner's anticipated reactions to a fixed sentence, constraint, and punishment when the discussion centres around the speaker's need to continue working at an emotionally exhausting job to repay a loan (Ritchie, 2004, 2009). Moreover, relevance theory does not address the issue of cultural aspects. Sperber and Wilson briefly mention that some of the concept characteristics are culturally constrained, but they do not make any further reference. Figurative meanings are often culturally determined (Charteris-Black, 2002; Semino, 2008), but if the meaning is closely related to mental representations and content, how would the mental representations of ad-hoc concept formation work for multilingual speakers? (See Section 4 for more details).

Another much-needed discussion in relevance theory would be the conventionalised metaphors and idiomatic expressions such as (4) face and hands of a clock or the (5) legs of a table which no longer have the power to strike as specifically figurative, and hence not needing a concept adjustment process. But the issue transcends the concept modulation. Research conducted by Cameron and Deignan (2006) shows that it is not only the lexical form that shapes the conventionality of metaphors, but also the conventionalisation of attitudinal judgments such as feelings, emotions and cultural aspect attached to them. Therefore, even the inferential comprehension of conventional metaphors should be further clarified when using the relevance-theoretic account in addition to a more recent account proposed by Carston (2010) to address more creative metaphors since feelings and cultural practices may play an equal important role as concept modulation when people comprehend metaphorical language. 


\section{Issues with the lexical pragmatic adjustment in relevance theory}

Concepts present a problem for the relevance-theoretic framework, especially when trying to account for metaphorical language. As briefly mentioned in the previous section, the relevance theoretical framework is heavily based on the lexical pragmatics paradigm which means that the comprehension process results from the processes of concept adjustment (through narrowing and broadening) to reflect specific meaning intended by the speakers.

Concepts in relevance theory are seen as representations that follow the Fodorian atomic view (they cannot be broken into constituents). The adjustment process is inferential, and it includes narrowing (e.g. (6) drink to refer to a more specific sense, i.e. alcoholic drink than the lexically encodedword of liquid) or broadening (e.g. (7) a ton of work used to communicate a more general sense of the word ton, or both at the same time (see Carston 2010, 2012), of the denotations of the encoded concept. The result is an adhoc concept created for specific instances which represents the base for the content of the utterance (its truth-conditional content). In opposition to the mapping account of metaphors presented by CMT, Sperber and Wilson's (2008) view places metaphorical comprehension in a continuum of cases ranging from literal expressions (e.g. legal texts) to category extensions, hyperboles and, at the more radical end of the spectrum, to metaphors. Yet, Carston (2010) points out that metaphors always require both narrowing and broadening of the lexically encoded concept. For example, in the metaphorical use of "boiling" in

(8) 'a creek is boiling over rocks'

the ad-hoc concept BOILING* results from the lexical concept BOILING being broadened and narrowed to remove the property of heat which defines BOILING. This phenomenon requires further explanation within the relevance theory framework because it challenges the continuity account of the relevance theoretic framework which places metaphor on a spectrum from a more literal meaning to language which is used more loosely such as metaphors, irony, hyperboles.

Furthermore, Sperber and Wilson's (2008) example

(9) He is a magician

clearly illustrates the formation of the occasion-specific concept, but at the same time, the utterance sheds some light on the perceived distinction between literal and metaphorical meaning. Sperber and Wilson claim that magician can mean literally someone who performs magic tricks to amuse an audience (MAGICIAN*) and metaphorically someone with supernatural powers who performs magic (MAGICIAN**). However, beyond the obvious distinction between the two meanings, some questions can still be asked about the two different senses. Can one meaning be more default than the other? If the 
answer is positive, then what would be the best way to distinguish between them? Additionally, what is the mundane conventionalized metaphorical sense of the above-mentioned example, considering that calling somebody a magician figuratively is similar to the norm of praising others for their skills?

The first implication of lexical pragmatics on the relevance theoretical account of metaphors is that the theory's perspective on concepts is somehow paradoxical. On the one hand, relevance theorists aim to minimise or even not recognise the literal-figurative distinction, but on the other hand, it relies heavily on concept adjustment, which, in short, and as simply as it is presented here, is the modification of the literal meaning, a process which involves using a lexical entry that corresponds to a mental representation to give rise to a logical form in the inferential process. Inferred ad-hoc concepts such as MAGICIAN* to refer to a skilled person in

(10) My chiropractor is a magician. You should go and see him (example taken from Sperber and Wilson, 2008)

are often, if not always, the modification of the literal meaning. That is although Sperber and Wilson (2015) strongly argue that people are regularly non-literal and literalness is not regularly preserved in communication.

Their alternative model of figurative language to the Gricean theory of conversational implicature (Grice, 1975) seems better suited to explain many communicative aspects, but further clarification needs to be made for literal and non-literal meaning. Sperber and Wilson (2015) clearly state that, contrary to Grice, understanding does not presuppose an initial literal interpretation and its rejection. Grice argued that when understanding metaphors, people would first reject literal meaning and then replace with non-literal interpretation, which means that the literal meanings of utterances were always accessed first. Despite that, Sperber and Wilson (2008) also seem to ascribe a somehow controlling aspect of the literal meaning in the creation of ad-hoc concepts since they strongly claim that meaning is constructed through narrowing and broadening processes. Interestingly, they claim that broadening literalness is not preserved in instances such as

(11) Holland is flat and

(12) (On a picnic, pointing to a rock): That's a table!, whereas in the narrowing process literalness can be preserved:

(13) Peter: Does Gerard like eating? Marry: He's French. (Sperber and Wilson, 2008).

But if literalness can act as a facilitator of meaning, that means it might have a greater role in communication than how relevance theorists present it. Also, is there a clear-cutting point between cases when it is and when it is not?

Meaning remains an important unresolved issue, especially in the debate of whether meaning constitutes a logical form. Relevance theory views literal meaning as an anchor with fixed characteristics which are used in 
lexical modulation. In other words, the relevance-theoretic assumption is that concepts as representations in people's minds act as a central interpretative hub. Furthermore, Sperber and Wilson (2008) do not consider how the lexicalisation process may occur for ad-hoc concepts. According to the theory, generally, the meaning constructed spontaneously is to achieve a goal-relevant in a given situation and with more encounters, the occasion-specific meaning becomes lexicalised. But if contextual features are also considered, how would one distinguish between an occasion-specific and a lexicalised concept? Additionally, if an occasion-specific concept becomes so striking that it would be remembered easily or linger in mind, would it be considered lexicalised? It is also worth bringing up the issue of why the encyclopaedic knowledge would not be considered concepts. In other words, there might be a possibility of concepts within a broader concept. For example, in

(14) He is a pig,

encyclopaedical information such as dirty is itself a concept. However, this might be a more relevant debate for questioning the mind overall, and not for a review of relevance theory. Recent research has started to point to more flexible nature of what has been known as concepts, to the point where concepts are no longer recognised as per-se representations (Allot and Textor, 2012, 2017). This paper supports the relevance-theoretic idea that there is a modification process in mental representations based on contextual and environmental factors, however, the present research questions the modulation process, especially the idea that there are cases when representations are modulated and cases when they are not.

Despite some ample support from studies including experiments to the idea that understanding a word in a given context may involve the construction of ad-hoc concepts or occasion-specific meanings of words' uses (Barsalou, 1983, 1999; Franks, 1995; Sanford and Stuart, 2002; see also Recanati, 2002; Glucksberg and Keysar 2002), more recent studies not only argue for a less promiscuous type of concepts, but they also call for a redefinition of concepts. Carston $(2010,2016)$ seems to focus on semantic ambiguity and calls for a redefinition of the notion of concepts within relevance theory. This seems to be much needed within the relevance-theoretic framework since cases of polysemy challenge the atomist approach to lexically encoded meaning (see Carston, 2015 for polysemous roots). Carston's (2016) argument that metaphorical comprehension results from both broadening and narrowing, whilst the comprehension process of similies does not, challenges the relevance-theoretic continuity view. The main reason for this lies in the relevance theoretic main continuity view which stipulates that metaphor is interpreted in the same way as other types of figurative language. Yet, if the process between metaphors and similies is not the same, it means that there are differences in understanding between metaphors and other linguistic 
meanings. It is not clear yet on Carston's or the relevance-theoretic account if other types of non-literal language require the same adjustments as metaphors.

However, an atomic view of concepts as presented in relevance theory does not seem to sustain the idea that the communicated content of a metaphorical expression is still recovered only via the encyclopaedic entries of the concepts. Carston $(2012,2013,2016)$ seems to move away from this definition in favour of a more schematic representation that is more in line with Allott and Textor's account (2012) where ad-hoc concepts are presented as clusters of information that are reflected in their activation (c.f. Recanati, 2004). For example, the ad-hoc concept SAINT* in

\section{(15) Sam is a saint}

is created by the information which is activated, and not created. Adopting a more radical perspective, Casasanto and Lupyan (2015) argue that all information is context-dependent (the context is always specified, although not always explicit) which makes all concepts and word meanings constructed as ad-hoc, in response to cues in context. Similarly, Recanati (2004) sees word meanings as memory traces which are activated in encounters with the world, and Barsalou (2005) looks at concepts as incomplete patterns to be filled in various occasions.

To address a clear problem in relevance theory, Carston (2012, 2015, 2016) envisages a non-conceptual view within relevance theory where conceptual expressions "point" to something looser than a stable conceptual space, a "template" for the construction of a fully propositional conceptual structure (c.f. Pustejovsky, 1995). In this schematic meaning view, words activate bundles of senses/concepts (polysemy) which are both fully or partially associated with them. That view seems to share similarities to how concepts are presented by Conceptual Metaphor Theory. The relationship between metaphor and simile as accounted in relevance theory raises more questions about concepts. Whilst the ad-hoc account is used for metaphors, it does not apply to similes. The comprehension process for

(16) My mother is like an angel includes a pragmatic process of inferring implicatures concerning how mother may resemble an angel, and not creating an unlexicalized category such as an ad-hoc concept. Here, relevance theory claims that the lexically encoded concept is preserved.

As Romero and Soria $(2010,2014)$ point out, ad-hoc concepts may emerge from mapping process which leads to the suggestion that relevance theory needs to revise their lexical pragmatics account of metaphorical processing. They consider that the difference between the mapping and the adhoc account is given by how the ad-hoc concept is constructed. Considering Wilson and Carston's (2006) atomic concepts view, then the result is a wider denotation. This seems problematic because, in her recent work, Carston (2010) points to more flexible nature of concepts than the one presented in 
relevance theory, a view which is not embraced by Romero and Soria (2014) who remain devoted to the conceptual domains. However, Wearing (2014) suggests analogical reasoning to help lexical processes, but only in category crossing and novel metaphors comprehension (where the resulting ad hoc concept is typically disjoint from the encoded concept - see the example above). She thinks that the analogical process is more effortful than the ad hoc creation and hence it relies on structural features obtained from both domains (i.e. in the metaphor's topic and vehicle). Nevertheless, the above suggestions are sought after to address the important shortcoming of the conceptual representations in the relevance-theoretical account of metaphors.

\section{Culture and meaning: beyond relevance}

As explained in the previous section, the notion of concept in relevance theory does not seem viable to interpret words and utterances. According to Allott and Textor (2017), standing meanings, encyclopaedic assumptions, and eventually ad hoc concepts are not felicitous to draw a mentally (psychologically) represented picture of words or utterances. This is because, for instance, the word (17) break can denote many properties and not only one.

(18) Break the glass

(19) Break the sentence

(20) Break the contract

However, other utterances (such as: 'they have a lifestyle which is very San Francisco', Wilson and Carston 2007, pp. 243) are said to be processed depending on 'general encyclopaedic knowledge' (Wilson and Carston 2007, pp. 243). By general encyclopaedic knowledge, Wilson and Carston (2007) refer to the social and the cultural knowledge hearers share. ${ }^{1}$

The role of context in relevance theory is indispensable. However, context according to relevance theory is dependent on the mental representation of assumptions that speakers produce for communication (Assimakopoulos, 2017). Context, here, seems to be restated by the assumptions speakers make. It is more cognitively reminiscent of what speakers think of the world. The implementation of context deviates from the social and the cultural traits, assuming that all speakers possess the same conceptualisation of the surrounding or share the same cultural assumptions. For instance, the example cited in Wilson and Carston paper (2007, pp. 243) 'the boy porched the newspaper, he did a napoleon for the camera, they have a lifestyle which is very San Francisco' can be confusing to learners of English as a second or foreign language. First of all, not many learners would be able to recognise a porch of a house. It costs more cognitive efforts to process the

\footnotetext{
${ }^{1}$ See the example of 'the boy porched the newspaper' (Wilson and Carston 2007: 13). As it is a culturally-dependent example that could be accessible to hearers sharing the same cultural traits.
} 
word 'porch' and create a concept of it that can be at risk of no felicitous context. $^{2}$

There is a high risk of developing completely different encyclopaedic assumptions (forming a concept) that can lead to ambiguity, irrelevance, and a clear violation of the principle of cognitive effects and efforts.

Applying this flaw to figurative aspects of speech, particularly idioms, can reveal more evidence of this. Considering the example (21) to make a pig of one's self in a scenario that two speakers of English met for a dinner in a restaurant (one is a native speaker, Peter and the other person is a learner of English as a second language, Ahmed).

(22) Peter: Ahmed's making a pig of himself!

Ahmed stops eating, looks at Peter and says: what do you mean?

Taking into account the relevance-theoretical accounts, example (22) communicates relevant inferential routes encoded in Peter's description of Ahmed through the idiomatic expression to make a pig of one's self. That is, and if only, both the speaker and the hearer (in the case of example (22), Peter and Ahmed share similar cultural assumptions. ${ }^{3}$ It can be imagined both a broadening and narrowing process of the concept pig into PIG*.

However, assuming a second scenario where Ahmed is on an English learning programme in London, but Peter is his flatmate who is originally from London. It is further confusing to image how broadening can take a place when two individuals from slightly different cultural orientations meet. Assuming that Ahmed is Algerian, where the utterance pig is very culturally inappropriate to be attributed to someone at whatever circumstances, lexical pragmatic adjustment can take a different turn from what the hearer intended to convey. The inferential assumptions drawn by Ahmed in (22) can lead to a break of communication. In other words, the communication is at the hazard of breaking down to an insult because there is no concept relatedness between what Peter intended and what Ahmed understood.

The following explanation details how example (22) can denote how relevant cognitive effects can be perceived under relevance satisfied or unsatisfied.

\footnotetext{
${ }^{2}$ Assuming, by plausibility, that only houses in the western world have a porch.

${ }^{3}$ Cultural assumptions are also differently casted. Cultural assumptions of a family living in London could be different from other families living in the same area. This is manifested in the difference between macro and micro-cultural assumptions.
} 


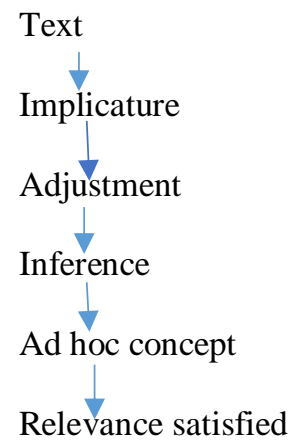

Figure 2: between-culture irrelevance processing

Example (22) highlights two situations, as explained earlier. Figure 1 shows the standard relevance-theoretic account of explaining figures of speech. According to Vega-Moreno (2007), the hearer in (22) would broaden the concept through pragmatic adjustments to enrich the concept of pig and guarantee the implication encoded by the speaker.

The relevance theoretic view that ...Every utterance, whether literally, loosely, hyperbolically or metaphorically intended, is processed using the same comprehension procedure, involving the same mutual adjustment process and guided by the more or less precise expectations of relevance (Vega-Moreno, 2007, pp. 124).

Relevance theory, however, does not provide a full theoretical account of how figurative aspects of speech (namely, idioms and metaphors) are processed with reference to cross-cultural communication. Considering figure 2, It can be seen how communication is interrupted at the level of ad hoc concepts inferred by Peter's attribute to Ahmed in (22). Because the inferential assumption derived from (22) is decoded accordingly to, merely, Ahmed's 
cultural assumptions about the utterance pig, a misunderstanding could take a place and therefore break the communication with relevance unsatisfied.

\subsection{Imperialist features of processing}

According to Goatly (1994), social context factors need to be acknowledged in processing inferential principles to establish a meaningful conversation between the speaker and the hearer. However, this criticism can also be extended to include relevance theory claims on understanding metaphors and idioms.

Going back to the example that Wilson and Carston used (the boy porched the newspaper), it can be assumed that this example takes a uniculturalism route to understand its implicatures. Having said that, VegaMoreno follows the same relevant theoretic account as she claims:

In interpreting an utterance containing an unfamiliar idiom, as in interpreting any other utterance, the hearer takes the encoded conceptual representation as to the starting point for inference. Following a path of least effort, he adds associated encyclopaedic assumptions to the context in order of accessibility, taking a particular inferential route whose output should be the range of implications the speaker might have intended to convey (Vega-Moreno, 2007, pp.196).

The relevance-theoretic approach to idiom comprehension, here, is perspicuously presented to felicitously explain first language speakers of English comprehension of idioms following a Unitarian ${ }^{4}$ approach to processing. How is example (22) understood according to the relevancetheoretic approach, assuming that Peter and Ahmed share completely different cultural assumptions about the word pig?

First, the novelty of figures to hearers is polemic. Accessibility, in this case, does not require least effort routes but rather encyclopaedic assumptions that are negotiated with the speaker (in the case of example 22, Ahmed stops eating, looks at Peter and says: what do you mean?). Second, there are two scenarios where the path of the least effort can be recognisably efficient, from our standpoints, one within familiar figurative aspects of speech and second with shared cultural schemas, which relevance theory implicitly addresses through relevance-theoretic accounts of comprehension.

Furthermore, Gibbs and Colston (2012) avow that different factors underline how figurative aspects of speech are understood where culture is an eminently distinguished factor. (23) More hideous than a pig is an Arabic

\footnotetext{
${ }^{4}$ An approach that considers only native speakers' cultural presuppositions in explaining metaphorical utterances comprehension.
} 
proverb which means that someone looks uglier than a pig. The concept of a $P I G^{*}$ in this example is very different from the one implied in (22). When different concepts are derived by speakers (Peter and Ahmed) who share ultimately different cultural traits, adjustments take different paths of what is usually intended by the speaker. Consequently, concepts developed also deviate from the contextual effects provided in the conversation ending at a break or a gap of communication with relevance unsatisfied.

Another example of metaphor, the body is a container for the emotion, is conceptualised differently according to Gibbs and Colston (2012). The embodiment of emotions in the body is perceived differently by various nationalities. How are concepts and adjustments formed when studying idioms and metaphors considering cross-cultural communication? Relevance theoretic account needs to consider culture as an efficient factor of interpretation or at least a factor that pushes the comprehension process to take certain different routes of what could be usually the case.

Therefore, there are some imperialist features of processing that seem to explore how 'John' and 'Peter' process figurative aspects of speech but not others who might share different cultural assumptions. The discussion and the exemplification of the theory are all biased toward western, English-speaking countries assumptions but arguing that the theory is universal. More recognition is needed to reflect upon other second and foreign English speakers' cultural assumptions in the theory and allow more variety (see Bouherar, $2017 \&$ Bouherar 2020a on how home cultures can be linked to idiom processing with a relevant theoretic account support).

\section{Alternatives for metaphorical comprehension}

Although there are other approaches to metaphorical figures processing which offer interesting explanations of the metaphorical phenomena, it would be almost impossible to combine their cognitive processing of metaphors, or even integrate elements into the relevance theoretic framework. For example, the conceptual metaphor theory, the direct access view, graded salience view, underspecification view (Gibbs and Colston, 2012) are alternative approaches to understand figurative aspects of speech. However, since this article explores aspects of relevance-theoretic accounts to understand metaphorical expressions, a requisite discussion of the pragmatic effects shaped by different cultural assumptions is needed.

Different referential processings happen at the level of cognitive comprehension of metaphorical expressions to satisfy the pragmatic effects intended by the speaker. Ultimately, this could not be possible regarding crosscultural communication between two or more individuals. More cognitive efforts are processed at the expense of a few cognitive effects, considering that speakers' cultural backgrounds are different. 
Considering the following example (24), it can be highlighted that the inadequacies caused by cultural assumptions which can jeopardise the conversation flow and ambiguate relevance.

(24) Paul: can't wait until the $5^{\text {th }}$ of November, after all last year fireworks were amazing. I wish I'll have similar atmosphere this year, old flames die hard!

Ahmed: it would be great if you tell me what is happening on the $5^{\text {th }}$ of November first!

Assuming that Paul lives in England, in (24) he is referring to Gay Fawkes' annual commemoration which takes a place on the $5^{\text {th }}$ of November each year. Keeping the same scenario of example (22), Ahmed is an English student. In (24), the hearer (in this case, Ahmed) builds loads of inferential assumptions to decode Paul's utterance. However, if you notice, two vague expressions need Ahmed's intention and cognitive efforts: one is the event of $5^{\text {th }}$ November and the second is the metaphorical expression of old flames die hard. There is a high risk that the conversation in (24) will not follow the least efforts path as claimed by Vega-Moreno (2007). Recognising the nature of the event can give the hearer access to the strings of the idiomatic expression's assumptions. The hearer cannot jump the line and process the second sentence of Paul, but rather he has to go through the first and then the second sentence due to the relative inferential routes that can result in decoding the second sentence.

Now, Ahmed seems to be in a position of familiarity with cultural assumptions and Paul in a position of unfamiliarity with Ahmed's communicated assumptions, assuming that Paul heard the idiomatic string for the first time and he is trying to search for relevance and possible inferential routes that give him access to the meaning.

(25) Ahmed: last year's Ramadan was exceptional, old flames die hard!

Paul: what is Ramadan?

Paul's question about Ramadan implies that he processed many inferential routes that, possibly, led him to nowhere as he ended up asking Ahmed about Ramadan. It can be argued also that Paul holds certain encyclopaedic assumptions and he is waiting to confirm or reject them, at least some of them. Without going further into the overall meaning of Ahmed's utterance, Paul's expectation of relevance was obstructed by the unfamiliarity of cultural assumptions of Ramadan. In order, the cognitive effects of Ramadan should be provided for Paul to proceed with the meaning of the idiomatic expression. 
Relevance theorists' efforts to explain the comprehension of figures of speech, particularly idioms and metaphors, need to be less rigid in the way it addresses mechanisms of understanding metaphorical utterances. Reading that hearers go through broadening and narrowing, least effort path, or expectation of relevance implies that both the speaker and the interlocutor are assumed to share similar cultural traits while they just need to adjust the language spoken through different apparatuses.

Moreover, the propositional analysis of examples used to explain aspects of relevance theory denote a sign of exclusivity of other cultures that uses English as a second language. For example, the use of the verb 'porch' in the boy porched the newspaper implies that this example is exclusive to native speakers of English.

Although the comprehensive attribute to the principle of relevance seems adequate, there could be no broadening or narrowing when processing metaphorical utterances unless both the speaker and the hearer share at least similar cultural assumptions. Within the frame of relevance-theoretic accounts, it is plain that native speakers' cultural assumptions played an evident role in explaining the sides of the theory in metaphor comprehension. However, considering the example (26) where both the speaker and the hearer share similar cultural backgrounds, the principle of relevance can be established, but inclusively compared to an exclusive manner that targets only native speakers.

(26) Mohamed: I can't forget those moments when we used to stay up late till morning on Ramadan nights, oh yeah! Old flames die hard (Bouherar, 2019, p. 297).

Ali: yeah, how can I forget that!

Ali inferential assumptions are directed toward the idiom old flames die hard and not Ramadan. More importantly, the utterance of Ramadan can give extra encyclopaedic assumptions to help Ali access the meaning of old flames die hard.

Even within-culture relevance, there are micro cultural assumptions (Bouherar, 2019). A person who lives in the east of Algeria for example shares slightly different cultural assumptions with another one who lives in the west of Algeria. Example (26) shows a macro-cultural assumption that is, arguably, known to the Muslim community. It is, however, about the practices of Ramadan that can vary from one place to another.

It is of a blatant claim to assert that culture can explain the relevancetheoretic account of metaphors compression, but rather it can unveil the idiosyncratic culture implicitly framed in relevance-theoretic routes of processing figures. Turning to culture in relevance theory with reference to figures, it has to be an inclusive term after all. It is, arguably, suggested that 
relevance theory can be extended to include, explicitly, discussions and explanations about the role of cultures (Bouherar, 2019, see also Bouherar 2020a) in metaphorical utterances comprehension with further empirical work.

\section{Conclusion}

In conclusion, this paper presented some flaws of relevance theoretic accounts of metaphorical comprehension. A review of the terminology used in explaining metaphors and idioms within the account is much needed. Concepts, for instance, are to be an attribute of what can be possibly constructed and extended in mind and not strictly linked to the truthconditional of the utterance speakers make. A more extended concern of narrowing and broadening features of concepts within metaphorical utterances should also be looked at with the lens of an appraisal. The inextricable relationship between concepts and the lexical adjustment of narrowing and broadening processes results in narrowing and broadening dependent on the definition of concept which does not offer much flexibility especially for nonpropositional elements, but, most importantly, is no longer a viable explanation of meaning in general. Even if the lexical adjustment processes might characterise meaning, this paper argues that the origin might not be a static concept as in relevance theory and these changes might not apply in some instances of communication for second language speakers. In other words, the article has pointed out that lexical adjustment, if the case, might be possible only in some cases where both the speaker and the hearer share similar cultural assumptions.

The relevance theoretic account did not provide details on how metaphorical utterances are understood under the use of cultural presuppositions. This paper distinguished between within-cultural relevance comprehension of figures of speech and between-cultural irrelevance comprehension, stating that relevance seems to be disrupted more in betweencultural irrelevance inferences. Additionally, this paper suggests, but does not provide alternatives, that relevance-theoretic account to explain metaphorical utterances, arguably, needs to broaden the aspects of relevance in comprehension. More research is needed to pave the road toward this proposal as this article just pointed to the fields of possible enrichment.

\section{References:}

1. Allott, N. and Textor, M. (2012). Lexical pragmatic adjustment and ad hoc concepts. International Review of Pragmatics, 4(2), 185-208

2. Allott, N. and Textor, M. (2017) Lexical modulation without concept. Dialectica, 71(3), 399-424. 
3. Assimakopoulos, S. (2017) Context in relevance theory. In: Blochowiak, J, Grisot, G., Durrleman, S. and Laenzlinger, C. (eds.) Formal models in the study of language: applications in interdisciplinary contexts. Springer: Gewerbestrasse, 221-242.

4. Barsalou, L. W. (1983) "Ad Hoc Categories.” Memory and Cognition 11, 211-27.

5. Barsalou, L. W. (1999) Perceptual symbol systems. The Behavioral and Brain Sciences, 22(4), 577-609.

6. Barsalou, L. W. (2005). Abstraction as Dynamic Interpretation in Perceptual Symbol Systems. In L. Gershkoff-Stowe and D. H. Rakison (Eds.), Carnegie Mellon Symposia on cognition. Building object categories in developmental time, 389-431. Lawrence Erlbaum Associates Publishers.

7. Bouherar, S. (2017) Idiom understanding and teaching: do we need to assume a target culture? Theory and Practice in Language Studies, 7(11), 943-951.

8. Bouherar, S. (2019) Understanding and teaching idioms: arguments for assuming home cultural contexts in teaching materials. $\mathrm{PhD}$ thesis. Middlesex University, London: 297.

9. Bouherar, S. (2020a) Home cultures and idiom processing: a shot cut to encyclopaedic assumptions of idioms. European Scientific Journal, 16(8), 31-61.

10. Bowdle, B. and Gentner, D. (2005) The career of metaphor. Psychological Review, 193-216.

11. Cameron, L., and Deignan, A. (2006) The emergence of metaphor in discourse. Applied Linguistics, 27 (4), 671-690.

12. Carston, R. (2002) Metaphor, ad hoc concepts and word meaning more questions than answers. UCL Working Papers in Linguistics, 14, $83-105$.

13. Carston, R. (2010) Lexical pragmatics, ad hoc concepts and metaphor: A Relevance Theory perspective. Italian Journal of Linguistics, 22 (1), 153-180.

14. Carston, R. (2010) Metaphor: Ad hoc concepts, literal meaning and mental imagery. Proceedings of the Aristotelian Society, 295 - 321.

15. Carston, R. (2012) Metaphor and the literal/nonliteral distinction. In K. a. Allan, The Cambridge Handbook of Pragmatics (pp. 469--492). Cambridge: Cambridge University Press.

16. Carston, R. (2015) Contextual adjustment of meaning. In: Riemer, N. (ed.) The Routledge handbook of semantics. London: Routledge, 195210.

17. Carston, R. (2016) The heterogeneity of procedural meaning, Lingua, 154-166 
18. Carston, R. and Hall, A. (2012) Implicature and explicature. In Schmid, H. J. (ed.), Cognitive Pragmatics. 4. Mouton de Gruyter. 4784.

19. Carston, R. and Wearing, C. (2011) Metaphor, hyperbole and simile: A pragmatic approach. Language and Cognition, 283-312.

20. Casasanto, D., and Lupyan, G. (2015) All concepts are ad hoc concepts. In Margolis, E. and Laurence, S. (Eds.), Concepts: New directions. 543-566, Cambridge, MA: MIT Press

21. Charteris-Black, J. (2002) Second language figurative proficiency: A comparative study of Malay and English. Applied Linguistics, 23, 104-133.

22. Clark, B. (2013) Relevance theory. Cambridge: Cambridge University Press.

23. Croft, W. (2009) Toward a social cognitive linguistics. New Directions in Cognitive Linguistics, ed. Vyvyan Evans and StŽphanie Pourcel, 395-420. Amsterdam: John Benjamins.

24. Deignan, A. (2008) Corpus linguistics and metaphor. In R. W. Gibbs, The Cambridge Handbook of Metaphor and Thought (pp. 280-294). Cambridge University Press.

25. Dunbar, R. I. M. (2004) Gossip in evolutionary perspective. Review of General Psychology, 8 (2): 100 - 110.

26. Fodor, J. (1998) Concepts: Where Cognitive Science Went Wrong, New York: Oxford University Press.

27. Fodor, J. (2000) The Mind Doesn't Work That Way, Cambridge, MA: MIT Press.

28. Franks, B. (1995) Sense Generation: A "quasi-classical" approach to concepts and concept combination. Cognitive Science 19:441-505.

29. Gibbs, R. W. and Colston, H. L. (2012) Interpreting figurative meaning. Cambridge University Press, Cambridge.

30. Glucksberg, S. and Keysar, B. (1990) Understanding metaphorical comparison - beyond similarity, Psychological Review, 1, 3-18.

31. Glucksberg, S. and Keysar, B. (1993) "How Metaphors Work." Metaphor and Thought. 2nd ed. Ed. Ortony, A. Cambridge: Cambridge 401-424.

32. Goatly, A. (1994) Register and the redemption of relevance theory: the case of metaphor. Pragmatics 4 (2): 139-181.

33. Golding, A. (2015) Mental imagery and metaphor. Letrônica, 8 (1), 20-32

34. Grice, H.P. (1975) Logic and conversation. In: Cole, P., Morgan, J. (Eds.), Syntax and Semantics, vol. 3. Academic Press, New York, 4158. 
35. Lakoff, G. and Johnson, M. (1980) Metaphors we live by. Chicago: University Press.

36. Newsome, M. R., and Glucksberg, S. (2002) Older adults filter irrelevant information during metaphor comprehension. Exp. Aging Res. 28, 253-267.

37. Pustejovsky, J. (1995) The Generative Lexicon. Cambridge MA: MIT Press.

38. Recanati, F. (2002) 'Unarticulated Constituents'. Linguistics and Philosophy 25, 299-345

39. Recanati, F. (2004) Literal Meaning. Cambridge: Cambridge University Press.

40. Ritchie, D. (2004) Lost in "conceptual spaces": Metaphors of conceptual Integration. Metaphor and Symbol. 19 (1), 31-50.

41. Ritchie, D. (2009) Relevance and Simulation in Metaphor. Metaphor and Symbol, 249-72.

42. Romero, E and Soria, B (2010) Eds. Explicit communication; Robyn Carston's Pragmatics. Palgrave Macmillan.

43. Romero, E. and Soria, B. (2014) Relevance Theory and Metaphor. Linguagem em (Dis)curso, 489-509.

44. Sanford, A. J. and Stuart, P. (2002) Depth of processing in language comprehension. Not noticing the evidence. Trends in Cognitive Sciences, 6 (9), $382-386$.

45. Semino, E. (2008) Metaphor in Discourse. Cambridge, New York: Cambridge University Press.

46. Sperber, D. and Wilson, D. (1995) Postface to the second edition of Relevance: Communication and Cognition. Oxford: Blackwell.

47. Sperber, D. and Wilson, D. (2002) Pragmatics, modularity and mindreading. Mind and Language, 17, 3-23.

48. Sperber, D. and Wilson, D. (2008) A deflationary account of metaphors. In: Gibbs, R.W. (Ed.), Cambridge Handbook of Metaphor and Thought. Cambridge University Press, Cambridge: 84-105.

49. Sperber, D. and Wilson, D. (2015) "Beyond speaker's meaning." Croatian Journal of Philosophy XV(44): 117-149.

50. Steen, G. (2011) The contemporary theory of metaphor-now new and improved! Review of Cognitive Linguistics, 9, 26-64.

51. Vega-Moreno, R. (2004) Metaphor interpretation and emergence. UCL Working Papers in Linguistics 16, 297-322.

52. Vega-Moreno, R. (2005) Idioms, transparency and pragmatic inference. UCL Working Papers in Linguistics 17, 389-425.

53. Vega-Moreno, R. (2005) Creativity and Convention: The Pragmatics of Everyday Figurative Speech. $\mathrm{PhD}$ thesis, University of London. 
54. Vega-Moreno, R. (2007) Creativity and convention: the pragmatics of everyday figurative speech. John Benjamins Publishing Company, Amsterdam.

55. Wearing, C. (2014) Interpreting novel metaphors. International Review of Pragmatics. 6, 78-102.

56. Wilson, D. and Carston, R. (2006) Metaphor, relevance and the 'emergent property' issue. Mind and Language, 21(3), 404-433.

57. Wilson, D. and Carston, R. (2007) A unitary approach to lexical pragmatics: relevance, inference and ad hoc concepts. In: BurtonRoberts, N. (Ed.), Pragmatics. Palgrave Macmillan, Basingstoke: $230-260$.

58. Wilson, D. and Sperber, D. (2004) Relevance Theory. In L.A. Ed. by Horn, The Handbook of Pragmatics, pp. 607-632. Oxford: Blackwell. 